\title{
Humanlike robots as platforms for Electroactive Polymers (EAP)
}

\section{Yoseph Bar-Cohen}

Yoseph Bar-Cohen, "Humanlike robots as platforms for Electroactive Polymers (EAP)," Proc. SPIE 6927, Electroactive Polymer Actuators and Devices (EAPAD) 2008, 692703 (29 April 2008); doi: 10.1117/12.776471

Event: SPIE Smart Structures and Materials + Nondestructive Evaluation and Health Monitoring, 2008, San Diego, California, United States 


\title{
Humanlike robots as platforms for Electroactive Polymers (EAP)
}

\author{
Yoseph Bar-Cohen \\ Jet Propulsion Lab, California Institute of Technology \\ 4800 Oak Grove Drive, Pasadena, CA 91109-8099 \\ yosi@jpl.nasa.gov, web: http://ndeaa.jpl.nasa.gov
}

\begin{abstract}
Human-like robots, which have been a science fiction for many years, are increasingly becoming an engineering reality thanks to many technology advances in recent years. Humans have always sought to imitate the human appearance, functions and intelligence and as the capability progresses they may become our household appliance or even companion. Biomimetic technologies are increasingly becoming common tools to support the development of such robots. As artificial muscles, electroactive polymers (EAP) are offering important actuation capability for making such machines lifelike. The current limitations of EAP are hampering the possibilities that can be adapted in such robots but progress is continually being made. As opposed to other human made machines and devices, this technology raises various questions and concerns that need to be addressed. These include the need to prevent accidents, deliberate harm, or their use in crimes. In this paper the state-of-the-art and the challenges will be reviewed.
\end{abstract}

Keywords: Biomimetics, biologically inspired technologies, robotics, EAP, electroactive polymers

\section{INTRODUCTION}

Nature has always provided humans a model for mimicking and inspiration [Bar-Cohen, 2005]. The value of Nature's inventions as a model for innovation was well recognized and the emergence of new technologies and capabilities are enabling development of biologicallt mimicked capabilities at accelerated rates. Making a human in an artificial form is the ultimate challenge to the field of biological inspiration and mimicking, which is also known as biomimetics. Humanlike robots are primarily machines that perform functions and tasks. However, if their capability is advanced to the point that turns them to become very smart with self-identity and cognitive behavior they will not be just another tool anymore. One may want to take into consideration that even though these robots still have many limitations the progress that we see today is mostly based on fifty years of advances since intelligent machines started emerging. With this in mind, the capability of the commercially available robots is showing quite impressive advances [Bar-Cohen and Hanson, 2008].

Currently, the most popular robot that already became an appliance in many homes is the vacuum cleaner called Roomba (made by iRobot). This robot, which has now more than two million users worldwide, is a disk shape device that vacuums the floor while traveling from one end of the house to the other avoiding obstacles and staying within the defined bounds. The effort to produce robots that are shaped and perform like humans has been going on for many decades and, initially, the produced robots were quite limited in what they could do and they had very much machinelike appearance. Advances in this technology led to robots that are significantly more lifelike than those preconceptions. Recently, efforts have surged to develop the technology into worldwide commercial products led to various robots with human appearance characteristics, called humanoids. Examples of such robots include Asimo (made by Honda), HOAP robots (made by Fujitsu), and the Partner Robots (made by Toyota). Further, humanlike robots as toys have already been commercialized and gained significant popularity. These tops include the Hasbro's Baby Alive doll and the Mattel's Miracle Moves Baby doll, which are able to move and exhibit functions that look like a real baby.

For commercial products, humanoid as the Honda's ASIMO are made with helmet-like head and do not have facial features except possibly for cameras that resemble eyes. The capabilities of these robots are still limited and their being "power-hungry" restrict their operation range and duration of usage since the current batteries need to be recharged quite often. However, the developed robots are used to determine their viability and to pave the way for future wide-spread commercialization of the technology. The market that these robots are expected to generate is estimated to reach billions of dollars. More specifically, the Japan Robot Association predicts that by 2025 the personal robot industry will be worth worldwide about $\$ 50$ billion a year. Also, South Korea has set an official goal of seeing robots in every home by

Electroactive Polymer Actuators and Devices (EAPAD) 2008, edited by Yoseph Bar-Cohen

Proc. of SPIE Vol. 6927, 692703, (2008) · 0277-786X/08/\$18 - doi: 10.1117/12.776471 
2013. Once these robots are developed to fill a niche application they will increasingly became part of our homes and offices. To help making these robots widely recognized and increase their interaction with humans, in celebration of the $50^{\text {th }}$ anniversary of Disneyland (June 2005), an ASIMO robot became a resident robotic entertainer of the visitors to the Honda ASIMO Theater, inside the Disneyland Park in Anaheim, Californian, USA. The capabilities of the Asimo robots are impressive and include climbing stairs, walking forward and backward, jogging, turning smoothly without pausing, and maintaining balance while walking on uneven slopes and surfaces. Also, it can grasp objects, switch lights on and off as well as open and close doors.

Robots that have humanlike appearance are mostly being developed at academic and research institutes. Shaping and operating a humanlike robot is quite a challenge to roboticists. Following the Uncanny Valley Hypothesis [see discussion in Chapters 5 and 7 at Bar-Cohen and Hanson, 2008], we are expected initially to be forgiving with the limitation of its humanlike appearance and performance. However, the closer these robots will be made to look and behave like humans the more critical we will most likely be towards their deficiencies. Since the functions of the developed robots are still far from the capability of biological creatures, some of the commercial robots are being shaped not to look like existing creatures and the Roboreptile (manufacturer by WowWee), which is an interactive toy robot, is an example of this approach. This choice of robot shape eliminates the concern that kids will compare their toy's behavior an appearance to a natural example.

In recent years, there are many types of humanoids and humanlike robots that were developed in many countries worldwide and various potential applications were considered, including health-care, entertainment, military, homeland defense (e.g., disabling bombs) and many others [Bar-Cohen and Hanson, 2008]. Specifically, the reported number of humanlike robots has increased enormously in such countries as Japan and Korea but researchers and engineers in other counties contributed to this trend too, including the USA, China, Russia, Australia, Germany, England, Italy, Spain and many others. In Japan, besides economical factors, the effort to make humanlike robots is motivated by the significant reduction in population resulting from their record low birthrate and from their having the longest lifespan of any nation with a large aging population. With the second-largest economy in the world, employers in Japan have great concerns regarding their future ability to fill jobs that require low-level skills and that may be dirty, dangerous, and physically demanding. For elderly, disabled or patients in rehabilitation, these robots may provide monitoring assistance 24-hours 7-days a week. A futuristic illustration of the possibility of having a humanlike robot accompany and monitoring an elderly person is shown in Figure 1. The head of the robot shown in this Figure was made with the recognizable image of the Michelangelo's statue, David, to reflect an object with the appearance of strength, youth and wisdom is transformed into a lifelike smart machine. The body was chosen to appear as a robot in order to make it clearly identifiable as a machine. As the development in this technology becomes more successful it will be increasingly important to distinguish them from organic creatures. Robots that appear as lifelike humans are already being made to appear like and operate as receptionists, guards, hospital workers, guides, and more. These robots were made to speak in various languages, and to perform various physical activities including dancing to music, playing musical instruments and even opening ceremonies.

FIGURE 1: A futuristic vision of using a humanlike robot to care and accompany elderly. Such robots may allow elderly to continue living in their own home while being monitored and helped in case of emergency or need for immediate treatment. Courtesy of the Graphics Artist Adi Marom.

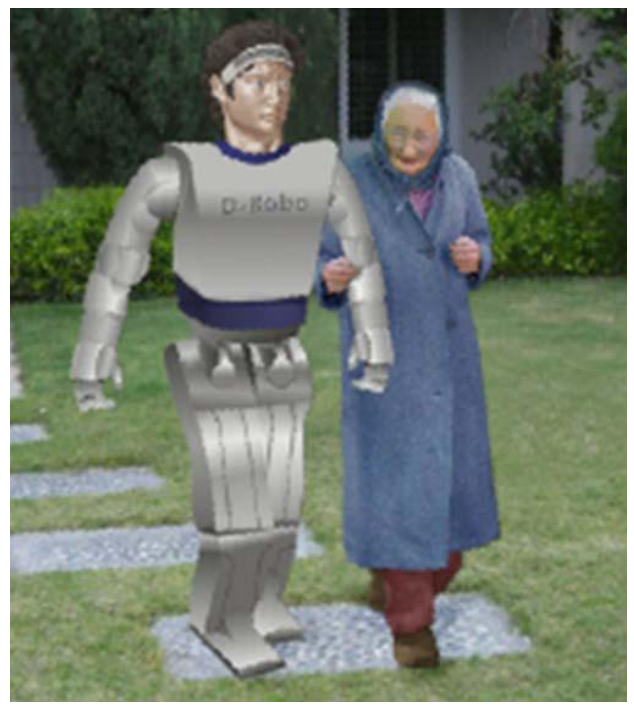


Humanlike robots are enjoying rapid advances in their capability, but there are still issues that limit their widespread use, including the very high cost of the sophisticated ones, their relatively short battery life, and their limited functionality. The ability to comprehend speech is still far from a level of a full conversation and they are still capable of discussing only predetermined subjects or topics. To make them affordable their prices will need to come down significantly and this will require reaching mass production levels and standardization of their operating system and parts. As robots become more useful and safe to operate, they will increasingly be used as a household helper, possibly replacing nannies and healthcare personnel, or acting as providers of other human-related services. Robots promise exciting future possibilities but the developers increasingly recognize that they need to be careful with the issues and hazards that their use may pose. Some of the issues that require attention include the need to prevent accidents, deliberate harm, or their use to perform unlawful tasks.

\section{CONCERNS RELATED TO THE DEVELOPMENT OF HUMANLIKE ROBOTS}

Advances in making humanlike robots will eventually enable them to perform more critical tasks and become more effective household appliances or even companions. Potentially, just as the development of the internet was benefited, making these robots as providers of sex services may lead to a large market and to rapid development. Such applications and many others that will result from the realization of truly humanlike machines are expected to raise ethical questions and concerns [see Chapter 7 in Bar-Cohen and Hanson, 2008]. As opposed to other machines that improve our lives, this technology will also complicate our lives or, if we are not sufficiently careful, may even hurt us. Beside the potential accidents, deliberate harm, or use of robots to commit crimes, they may pose danger due to their being given direct access to our intimate and confidential information and possibly making it public or using the information against us. Addressing these issues and concerns require careful attention from the developers and the companies that will bring the robots to the market.

Science fiction movies, literature, and homepages on the Internet are portraying a negative image of the potential dangers of developing humanlike robots. While we may be many years away from realizing such fictional capabilities there are realistic possibilities that will need to be addressed as robots evolve. Efforts by robotic researchers are already underway to study the possible issues of concern, and to find ways to address them by establishing codes of ethics, guidelines and algorithms for ethical, friendly AI to be used to make robots. To allow maintaining the freedom to develop such robots, these efforts are important for the developers of the technology because otherwise laws may be imposed on researchers to limit or prohibit development of humanlike robots. For this purpose, researchers are seeking to recommend boundaries for human-robot interactions before super-intelligent robots become capable beyond our control. The emphasis is on ensuring that humans maintain control of robots, prevent their illegal use, protect the data that robots acquire, and establish methods of clearly identifying and tracing such robots. More than just imposing external limits on robots, it is important to consider how to make friendly AI algorithms that will be installed in intelligent humanlike robots to make them ethical at the core of their minds.

Since these concerns involve robots achieving something like human-level intelligence, the fear of such robots becoming a threat to humanity is likely decades away from becoming a serious one. This doesn't mean that we shouldn't bother considering this issue now. In fact, now may be just the time to take action, adjusting the way our robots are built to prevent them from being sociopathic and to make sure that they are helpful, and good.

\section{HUMANLIKE ROBOTS DEVELOPMENT CHALLENGES}

Humanlike robots are still far from emulating the full capability of a real person even though [see Chapter 2 in Bar-Cohen and Hanson, 2008], some of the developed robots were made to look very much like a human and have great capability. The currently developed robots are able to perform relatively few functions and they are designed mostly to execute specific tasks. The use of AI is already enabling facial recognition and building robots with personality and behavioral differences between the produced duplicates of the particular 
robots. In spite of the progress, there are still many challenges to the capability of humanlike robots. These include making a robot that can conduct a comprehensive conversation with human on a broad range of subjects, walking fast in a crowd without hitting anyone, and operating over an extended period of time without the need to recharge its batteries.

Generally, to develop low cost robots in large production volume there is a need for standard hardware and software platforms that will have inter-changeability and compatibility as in personal computers. Thus, designers will not need to start from scratch each time they develop a new model of robot. Moreover, there is a need to increase the response speed and the reaction to changes in the environment. This will require the use of many miniature light weight sensors with distributed processing capability as well as fast high power density actuators. The use of networked wireless robots can benefit them from the power of personal computers to handle complex tasks, including image recognition, navigation, and the avoidance of collision. Further, there is a need for advances in automated design and prototyping from macro scale locomotion to micro scale sensing, actuation and drive electronics.

\section{EAP ACTUATORS AS ARTIFICIAL MUSCLES}

Actuators are used as emulators of muscles and are responsible for the movement and mobility of robots and their appendages. The types of actuators that are generally used include: electric, pneumatic, hydraulic, piezoelectric, shape memory alloys or ultrasonic motors. Motors are widely used to move components of humanlike robots but they behave differently than our natural muscles and have a totally different operation mechanism. Specifically, DC and AC type electromagnetic motors are used with gears to compromise speed of rotation for torque capability. In contrast to motors, natural muscles are both compliant (flexible or rubbery) and exhibit linear behavior [Full and Meijer, 2004]. These capabilities are important to emulate since they address key control requirements for robotic applications. The closest to have the potential to emulate natural muscles are the electroactive polymers (EAP) that have emerged in recent years and gained the name "artificial muscles" [Bar-Cohen., 2004; Hanson, 2004]. In the 1990s, the developed materials were very weak in terms of the ability to move or lift objects. Recognizing the need for international cooperation among the developers, users, and potential sponsors, the author initiated and organized in March 1999 the first annual EAPAD Conference. At the opening of this conference, he posed a challenge to the worldwide researchers and engineers to develop a robotic arm that is actuated by artificial muscles to win an armwrestling match against a human opponent (http://ndeaa.jpl.nasa.gov/nasa-nde/lommas/eap/EAP-armwrestling.htm).

In 2005, three groups that consisted of scientists and engineering students were ready with EAP actuated robotic arms for the first armwrestling match against a human. The contest was held on March 7, 2005, at San Diego, CA, as part of this annual SPIE's EAPAD Conference and it was organized with assistance from the United States ArmSports who provided the wrestling table and a representative as one of the contest judges. The human opponent was a 17-years old straight-A high school female student from San Diego [http://ndeaa.jpl.nasa.gov/nasa-nde/lommas/eap/amerah/thehuman-opponent.htm], whose name is Panna Felsen. In Figure 2, a photo shows a member of the group of students from Virginia Tech preparing for one of the three wrestling matches that were held. Even though the 17-old female student, won in all the three wrestling matches, this has been a very important milestone for the field.

Since the currently developed robotic arms with EAP actuators are not at a level that allows winning against humans, the author changed the focus of the near future contents. As of 2006, the contest was turned to measuring the arms capability and comparing the data of the competing arms. A fixture was made jointly by individuals from UCLA and the author's group at JPL (see Figure 3) allowing for measuring the speed and pulling force capability of the arms. The test fixture was equipped with a pulling cable that was connected on the fixture side to a force gauge. To gauge the speed a $0.5-\mathrm{kg}$ weight was mounted on the cable which has to be lifted to the top of the fixture and the time to reach the top is measured. To establish a human reference data, Panna Felsen was invited to the event and her capability was measured. The 2006 results of three participating arms have shown about two orders of magnitude slower and weaker force capability than the student. In a future conference, once advances in developing such arms reach sufficiently high level, a professional wrestler will be invited for the next human/machine wrestling match. 
FIGURE 2: The robotic arm driven by artificial muscles, made by Virginia Tech students, is being prepared for the match against the human opponent, Panna Felsen, a 17-years old student from San Diego.

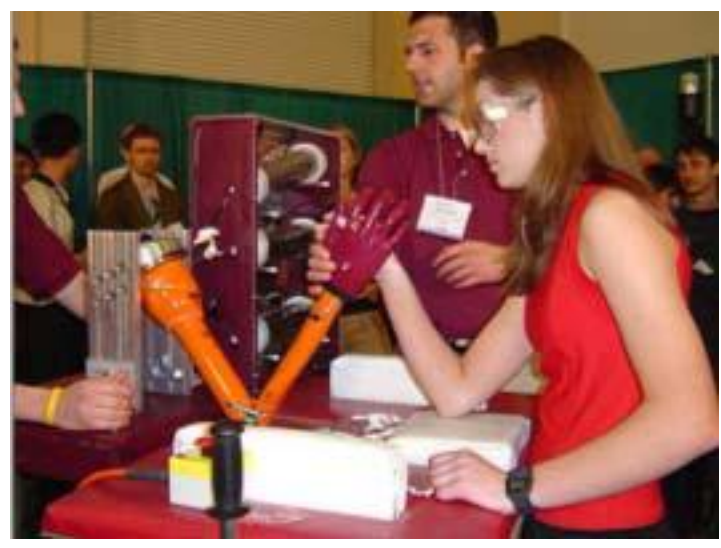

FIGURE 3: The fixture that is used to test the force and speed of the EAP actuated robotic arms

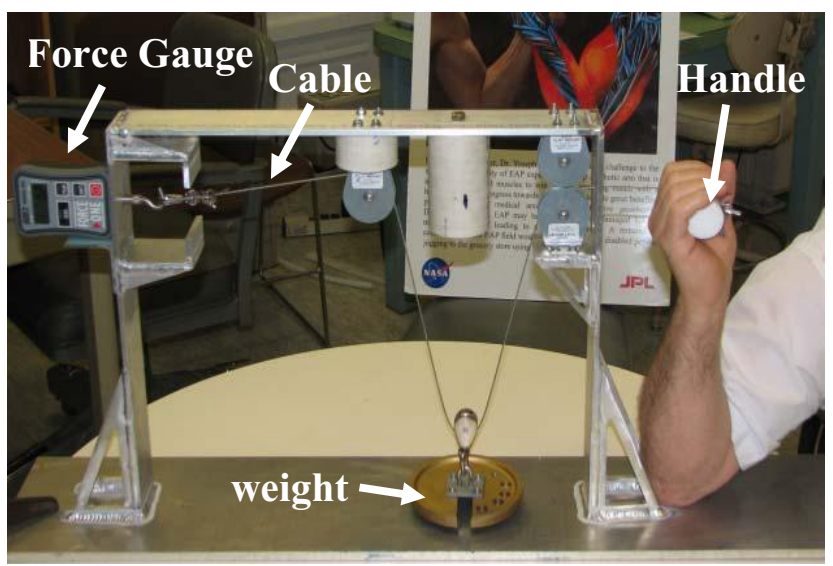

\section{CONCLUSION}

Emulating the human appearance, functions and intelligence is the ultimate goal of humans' efforts to reproduce ourselves in art and technology. Making humanlike robots involves developing machines that are the copy of human appearance and imitating our behavior as biological systems. Increasingly, such robots are becoming a reality as a result of recent technology advances [Bar-Cohen and Breazeal, 2003; Bar-Cohen 2005]. Tools such as finite element modeling, computer simulations, rapid image processing, graphic displays and animated simulations, as well as many others are allowing for making enormous progress towards producing life-like robots. There are still numerous challenges to producing humanlike robot. To overcome these challenges requires multidisciplinary expertise that includes engineering, computational and material science, robotics, neuroscience, and biomechanics. This development need to be supported by progress in many related fields, including artificial intelligence, artificial muscles, artificial vision, speech synthesizers, mobility, control, and many others. As these robots become more useful in our life they may start appearing as a household appliance or our companions and probably a common sight in our future environment.

Generally, many science fiction movies have generated a horrible image of the possible consequences of having humanlike robots go out of control. While these are science fiction scenarios there are realistic concerns of this technology and roboticists are currently making efforts to establish codes of ethics and guidelines to deal with the required action.

\section{ACKNOWLEDGEMENT}

Some of the research reported in this manuscript was conducted at the Jet Propulsion Laboratory (JPL), California Institute of Technology, under a contract with the National Aeronautics and Space Administration (NASA). The author would like to thank David Hanson, Hanson Robotics, Inc., for his technical suggestions, and to Adi Marom, Graphics Artist, for her futuristic illustration. 


\section{REFERENCES}

Bar-Cohen Y., and C. Breazeal (Eds.), "Biologically-Inspired Intelligent Robots," SPIE Press, Bellingham, Washington, Vol. PM122, ISBN 0-8194-4872-9 (May 2003), pp. 1-393.

Bar-Cohen Y. (Ed.), "Electroactive Polymer (EAP) Actuators as Artificial Muscles - Reality, Potential and Challenges," 2nd Edition, ISBN 0-8194-5297-1, SPIE Press, Bellingham, Washington, Vol. PM136, (March 2004), pp. 1-765

Bar-Cohen Y., (Ed.), "Biomimetics - Biologically Inspired Technologies," CRC Press, Boca Raton, FL, ISBN 0849331633, (November 2005), pp. 1-527.

Bar-Cohen Y., and D. Hanson, "Humanlike Robots - The engineering realization and implications of the science fiction in synthetic humans," This book is expected to be published in 2008.

Full R. J., and K. Meijir, "Metrics of Natural Muscle Function," Chapter 3 in [Bar-Cohen, 2004], pp. 73-89.

Hanson D., "Converging the Capability of EAP Artificial Muscles and the Requirements of Bio-Inspired Robotics," Proceedings of the SPIE EAP Actuators and Devices (EAPAD) Conf., Y. Bar-Cohen (Ed.), Vol. 5385, SPIE, Bellingham, Washington, (2004) pp. 29-40. 